\title{
Schur Lemma and the Spectral Mapping Formula
}

by

\author{
Antoni WAWRZYŃCZYK
}

Presented by Stanistaw WORONOWICZ

Summary. Let $B$ be a complex topological unital algebra. The left joint spectrum of a set $S \subset B$ is defined by the formula

$$
\sigma_{l}(S)=\left\{(\lambda(s))_{s \in S} \in \mathbb{C}^{S} \mid\{s-\lambda(s)\}_{s \in S} \text { generates a proper left ideal }\right\} .
$$

Using the Schur lemma and the Gelfand-Mazur theorem we prove that $\sigma_{l}(S)$ has the spectral mapping property for sets $S$ of pairwise commuting elements if

(i) $B$ is an m-convex algebra with all maximal left ideals closed, or

(ii) $B$ is a locally convex Waelbroeck algebra.

The right ideal version of this result is also valid.

Introduction. By a topological algebra we mean a topological vector space $B$ which is an algebra such that the product $B \times B \ni(x, y) \mapsto x y \in B$ is continuous.

We wish to find a possibly wide class of unital topological algebras $B$ whose left (or right) proper ideals have the following property:

For every left ideal I and every set $S$ of pairwise commuting elements such that $I S \subset I$ there exists a proper ideal $J$ such that $I \subset J$ and $S \subset J+\mathbb{C}$.

We call this property the projection property of the family of left ideals because it implies the projection property of the left joint spectrum in $B$ which is studied in Section 2. We consider there the concept of left joint spectrum $\sigma_{l}(S)$ for systems of pairwise commuting elements of $B$, also infinite ones.

The principal tools used in the proof of the projection property are the Schur lemma in its purely algebraic version and the Gelfand-Mazur theorem.

2000 Mathematics Subject Classification: Primary 46H10; Secondary 46H15, 46H30. mula.

Key words and phrases: Waelbroeck algebra, joint spectrum, spectral mapping for- 
The latter is applied to a quotient $A / M$, where $A$ is a subalgebra of $B$ and $M$ is a two-sided ideal in $A$ and a left ideal in $B$.

By the Gelfand-Mazur theorem we mean its general version obtained by applying the classical Banach algebra proof.

If $D$ is a complex topological division algebra with continuous inverse and with a nonzero continuous linear functional $\varphi$, then if there existed $a \in D \backslash \mathbb{C}$, the function $\mathbb{C} \ni \lambda \mapsto(a-\lambda)^{-1}$ would be an entire bounded function vanishing at infinity. By the Liouville theorem this function is identically zero, which is a contradiction. This implies $D \cong \mathbb{C}$.

Let $D^{\prime}$ denote the dual space of $D$. So, the version of the Gelfand-Mazur theorem used here is:

If $D$ is a complex topological division algebra with continuous inverse and such that $D^{\prime} \neq 0$ then $D \cong \mathbb{C}$.

Our principal problem is to impose reasonable conditions on $B$ ensuring the continuity of the inverse in $A / M$. The continuity of the inverse in $B$ is neither necessary nor sufficient for this end.

We show that the projection property for left (or right) ideals is valid if

(i) $B$ is an m-convex algebra with all maximal left (resp. right) ideals closed, or

(ii) $B$ is a locally convex Waelbroeck algebra.

In the case of a Banach algebra $B$ any one of the two versions provides a new proof of the projection property and the spectral mapping theorem of R. Harte [3]. For finitely generated ideals, case (ii) was proved in [4].

In Section 2 we deduce in a standard way the spectral mapping theorem for joint spectra $\sigma_{l}\left(\left\{x_{z}\right\}_{z \in Z}\right)$ of (possibly infinite) systems of pairwise commuting elements.

1. The projection property for left ideals. We begin with the purely algebraic part of the problem.

Denote by $B$ a complex algebra with unit $e$. Let $I$ be a left ideal in $B$ and let $E$ be a subalgebra of $B$ such that $I a \subset I$ for all $a \in E$ and $[a, b]=a b-b a \in I$ for all $a, b \in E$.

Consider the set $\mathfrak{I}$, ordered by inclusion, of all ideals $J$ in $B$ which satisfy $I \subset J$ and $J E \subset J$. By the Kuratowski-Zorn lemma there exists a maximal element $M$ in $\mathfrak{I}$.

Consider the quotient space $X=B / M$ with the natural action of $B$ given by the formula $l_{b}[x]=[b x]$ and with the action of the algebra $E$ defined by $r_{b}[x]=[x b]$. Finally, we define a representation of $B \times E$ in $X$ by

$$
T_{(b, c)}[x]=l_{b} r_{c}[x]=[b x c] .
$$

Proposition 1.1. The representation $T$ of $B \times E$ in $X$ is irreducible. 
Proof. Denote by $\pi: B \rightarrow X$ the natural projection. Let $V \subsetneq X$ be a $(B \times E)$-invariant vector space. In particular $V$ is $B$-invariant, so $J=\pi^{-1}(V)$ is a left ideal of $B$ containing $M$. The invariance of $V$ under the action of $E$ implies $J E \subset J$. By the maximality of $M$ it follows that $J=M$.

Denote by $D$ the commutant of the representation $T$, that is, the set of all linear operators in $X$ which commute with every operator $T_{(b, c)},(b, x) \in$ $B \times E$. The Schur lemma (see e.g. [1]) leads immediately to the following theorem.

TheOREM 1.2. $D$ is a division algebra.

The algebra $D$ can be described in terms of the algebra $B$. Set

$$
A=\{b \in B \mid M b \subset M \text { and } b x-x b \in M \text { for all } x \in E\} .
$$

It is clear that $A$ is a unital subalgebra of $B$ and $M$ is a two-sided ideal in $A$. A right action of $A$ in $X$ can be defined by the formula $r_{b}[x]=[x b]$. It is convenient to consider also a modified algebraic product in $A$. For $a, b \in A$ set $a \star b=b a$. The algebra $(A, \star)$ is denoted by $\mathcal{A}$, while $\mathcal{M}$ denotes $(M, \star)$.

THEOREM 1.3. Every element of $D$ is of the form $R[x]=[x b]$ for some $b \in \mathcal{A}$. Moreover $D \cong\left\{u \in B / M \mid l_{c} u=r_{c} u, \forall c \in E\right\} \cong \mathcal{A} / \mathcal{M}$.

Proof. Let $R \in D$. Observe that $R[x]=R l_{x}[e]=l_{x} R[e]$ for all $x \in B$. If we write $R[e]=[b]$ then for every $m \in M$ we obtain

$$
0=R[m]=l_{m}[b]=[m b],
$$

giving $M b \subset M$.

The operator $R$ commutes with all $r_{c}, c \in E$, whence

$$
[b c]=r_{c} R[e]=R r_{c}[e]=R[c]=l_{c}[b]=[c b],
$$

so $b \in \mathcal{A}$ and $u=[b]$ satisfies the condition $l_{c} u=r_{c} u$ for all $c \in E$.

All elements of $D$ are of the form $R_{[b]}[x]:=[x b]$ for some $b \in \mathcal{A}$. The map $\mathcal{A} \ni b \mapsto R_{[b]}$ is an algebra homomorphism because

$$
R_{[b \star c]}[x]=[x c b]=R_{[b]}[x c]=R_{[b]} R_{[c]}[x] .
$$

The kernel of this homomorphism consists of the elements of $\mathcal{M}$, so $D \cong$ $\mathcal{A} / \mathcal{M}$.

Suppose now that $B$ is a topological algebra. The results we are interested in rest on a theorem of Gelfand-Mazur type, hence the topology of the quotient $D \cong \mathcal{A} / \mathcal{M}$ is essential.

For an arbitrary set $S \subset \mathcal{A}$ we denote by $I_{l}(S)$ the left ideal in $\mathcal{A}$ generated by $S$.

THEOREM 1.4. Let $B$ be a complex unital m-convex algebra whose maximal left ideals are all closed. Then for every left ideal I of $B$ and every set 
$S \subset B$ such that $I S \subset I$ and $[S, S] \subset I$ there exists a maximal left ideal $M$ in $B$ and a function $S \ni c \mapsto \mu(c) \in \mathbb{C}$ such that $I+I_{l}\left(\{c-\mu(c)\}_{c \in S}\right) \subset M$.

Proof. We apply the above results in the case of $E$ equal to the subalgebra in $B$ generated by $S$. Since $[S, S] \subset I$, also $[E, E] \subset I$. Let $D \cong \mathcal{A} / \mathcal{M}$ be the corresponding division algebra. Let us provide $\mathcal{A}$ with the topology induced by $B$. The closure of $M$ satisfies $\bar{M} E \subset \bar{M}$, so $\bar{M}=M$ by the maximality of $M$. Clearly, $\mathcal{A}$ is closed in $B$, while $\mathcal{M}$ is closed in $\mathcal{A}$.

The algebra $D$ is also an m-convex algebra, so the inverse is continuous in it. By the version of the Gelfand-Mazur presented in the Introduction, $D \cong \mathbb{C}$.

The condition $[S, S] \subset I \subset M$ means that $S \subset \mathcal{A}$. For every $c \in S$ we have $R_{[c]} \in D$, so there exists $\mu(c) \in C$ such that $[x c]=\mu[x]$, which gives $x(c-\mu(c)) \in M$ for every $x \in B$.

In particular $I+I_{l}\left(\{c-\mu(c)\}_{c \in S}\right) \subset M$. By Propositon 1.2 the action of $B \times A$ on $X=B / M$ is irreducible. Since $A$ acts on $X$ multiplying by scalars, the action of $B$ on $X$ is irreducible, which means that $M$ is a maximal ideal of $B$.

As proved in [4], in locally convex Waelbroeck algebras the left ideals generated by a finite set of commuting elements also have the property described in the last theorem. An argument based on the Schur lemma allows us to generalize that result to arbitrary ideals, at the same time simplifying the proof.

THEOREM 1.5. Let $W$ be a locally convex Waelbroeck algebra. Then for every left ideal $I$ of $W$ and every set $S \subset W$ such that $I c \subset I$ for all $c \in S$ and $[S, S] \subset I$ there exists a maximal ideal $M$ in $B$ and a function $S \ni c \mapsto \mu(c) \in \mathbb{C}$ such that $I+I_{l}\left(\{c-\mu(c)\}_{c \in S}\right) \subset M$.

Proof. We apply the same method as in the proof of Theorem 1.4 to construct the ideal $M$ and the division algebra $D=\mathcal{A} / \mathcal{M}$. Let $a \in \mathcal{A}$ be invertible in $B$. Define $M^{\prime}=M+M a^{-1}$. Obviously $M^{\prime}$ is a left ideal of $W$. If $e \in M^{\prime}$, that is, if $e=m+m^{\prime} a^{-1}$ with $m, m^{\prime} \in M$, then $a=m a+m^{\prime} \in M$, which contradicts the invertibility of $a$. The ideal $M^{\prime}$ is a proper left ideal. We claim that $M^{\prime} \in \mathfrak{I}$. For every $b \in E$ there exists $m \in M$ such that $b a=a b+m$, hence

$$
\begin{aligned}
M^{\prime} b & =\left(M+M a^{-1}\right) b \subset M+M a^{-1} b a a^{-1}=M+M a^{-1}(a b+m) a^{-1} \\
& =M+M b+M a^{-1} m a^{-1} \subset M+M a^{-1}=M^{\prime} .
\end{aligned}
$$

By the maximality of $M$ in $\mathfrak{I}$ it follows that $M^{\prime}=M$, which implies that $M a^{-1} \subset M$. Moreover,

$$
a^{-1} b-b a^{-1}=a^{-1}(b a-a b) a^{-1}=a^{-1} m a^{-1} \in M,
$$

so finally $a^{-1} \in \mathcal{A}$. 
The algebra $\mathcal{A}$ is a subalgebra of $W$ closed under taking inverses. This implies that $\mathcal{A}$ is also a locally convex Waelbroeck algebra, as also is its quotient algebra $D=\mathcal{A} / \mathcal{N}$. In particular, the inverse is continuous in $D$, so by the Gelfand-Mazur theorem $D \cong \mathbb{C}$. We finish the proof exactly as for Theorem 1.4.

The right ideal versions of these results are valid as well.

The results proved in this section also lead to the following structural property of the algebras in question.

Corollary 1.6. Let $B$ be one of the algebras considered in Theorem 1.4 or Theorem 1.5. Let $A$ be a commutative subalgebra of $B$. Then there exists a maximal left (resp. right) ideal $M$ of $B$ such that $A \subset M+\mathbb{C}$.

Proof. If we take $I=\{0\}$, the conditions $I A \subset I$ and $[A, A] \subset I$ are satisfied. By Theorem 1.4 or Theorem 1.5 respectively, there exists a maximal ideal $M$ in $B$ such that for every $a \in A$ and an appropriate $\mu \in \mathbb{C}$ we have $a-\mu \in M$, which ends the proof.

\section{Left spectrum and spectral mapping theorems for commuta-} tive systems. The projection property investigated in the former section is closely related to the projection and spectral mapping properties of the left joint spectrum in $B$. Following the ideas from [5] we define the left spectrum for an arbitrary family of pairwise commuting elements of $B$.

Let $B$ be again a complex unital algebra. Let $Z$ be an arbitrary set. Denote by $\mathfrak{c}_{Z}(B)$ the set of all functions $\mathbf{x}_{Z}: Z \ni z \mapsto x_{z} \in B$ such that the elements $x_{z}$ pairwise commute.

The left joint spectrum of $\mathbf{x}_{Z}$ is the set $\sigma_{l}\left(\mathbf{x}_{Z}\right)$ of all functions $\lambda: Z \rightarrow \mathbb{C}$ such that $I_{l}\left(\left\{x_{z}-\lambda(z)\right\}_{z \in Z}\right)$ is a proper ideal in $B$.

For $Y \subset Z$ we define the natural projection

$$
\pi_{Y}^{Z}: \sigma_{l}\left(\mathbf{x}_{Z}\right) \rightarrow \sigma_{l}\left(\mathbf{x}_{Y}\right)
$$

by restricting $\lambda \in \mathbb{C}^{Z}$ to $Y$. Obviously $\pi_{Z}^{Y} \sigma_{l}\left(\mathbf{x}_{Z}\right) \subset \sigma_{l}\left(\mathbf{x}_{Y}\right)$. We also write $\pi_{Y}^{Z} \mathbf{x}_{Z}=\left.\mathbf{x}_{Z}\right|_{Y}$.

THEOREM 2.1. Let $B$ be an m-convex algebra with all maximal left ideals closed or a locally convex Waelbroeck algebra. Then for every $\mathbf{x}_{Z} \in \mathfrak{c}_{Z}(B)$ and every $Y \subset Z, \pi_{Y}^{Z} \sigma_{l}\left(\mathbf{x}_{Z}\right)=\sigma_{l}\left(\pi_{Y}^{Z} \mathbf{x}_{Z}\right)$.

Proof. It remains to prove that the projection $\pi_{Y}^{Z}: \sigma_{l}\left(\mathbf{x}_{Z}\right) \rightarrow \sigma_{l}\left(\mathbf{x}_{Y}\right)$ is onto. Let $\mu \in \sigma_{l}\left(\mathbf{x}_{Y}\right)$. Then $I_{l}\left(\left\{x_{z}-\mu(z)\right\}_{z \in Z}\right)$ is a proper ideal in $B$. Let $S=\left\{x_{z}\right\}_{z \in Z \backslash Y}$. By applying Theorem 1.4 or 1.5 we can construct a function $\mu^{\prime}: S \rightarrow \mathbb{C}$ such that $I+I_{l}\left(\left\{s-\mu^{\prime}(s)\right\}_{s \in S}\right)$ is a proper left ideal in $B$. Define a function $\lambda \in \mathbb{C}^{Z}$ equal to $\mu(z)$ for $z \in Y$ and to $\mu^{\prime}\left(x_{z}\right)$ if $z \in Z \backslash Y$. Then $\lambda \in \sigma_{l}\left(\mathbf{x}_{Z}\right)$ and $\pi_{Y}^{Z} \lambda=\mu$. 
Let $\mathbf{x}_{Z} \in \mathfrak{c}_{Z}(B)$ and $\mathbf{y}_{U} \in \mathfrak{c}_{U}(B)$. Let $P=\left\{p_{u}\right\}_{u \in U}$ be a collection of polynomials such that for every $u \in U$ there exist $z_{1}, \ldots, z_{m_{u}} \in Z$ such that $y_{u}=p_{u}\left(x_{z_{1}}, \ldots, x_{z_{m_{u}}}\right)$. We then say that $\mathbf{y}_{U}$ is a polynomial image of $\mathbf{x}_{Z}$ and we write $\mathbf{y}_{U}=P\left(\mathbf{x}_{Z}\right)$.

For $\lambda \in \sigma_{l}\left(\mathbf{x}_{Z}\right)$ we define $P(\lambda)(u)=p_{u}\left(\lambda\left(x_{z_{1}}\right), \ldots, \lambda\left(x_{z_{m_{u}}}\right)\right)$ obtaining an element of $\mathbb{C}^{U}$. Finally, we set $P\left(\sigma_{l}(S)\right)=\left\{P(\lambda) \mid \lambda \in \sigma_{l}(S)\right\}$.

Theorem 2.2. Let $B$ be as in Theorem 2.1 and let $\mathbf{x}_{Z} \in \mathfrak{c}_{Z}(B)$ and $\mathbf{y}_{U} \in \mathfrak{c}_{U}(B)$ be such that $\mathbf{y}_{U}=P\left(\mathbf{x}_{Z}\right)$ for some $P=\left\{p_{u}\right\}_{u \in U}$. Then the following spectral mapping formula is valid:

$$
P\left(\sigma_{l}\left(\mathbf{x}_{Z}\right)\right)=\sigma_{l}\left(P\left(\mathbf{x}_{Z}\right)\right) .
$$

Proof. The inclusion $P\left(\sigma_{l}\left(\mathbf{x}_{Z}\right)\right) \subset \sigma_{l}\left(P\left(\mathbf{x}_{Z}\right)\right)$, called the one-way spectral mapping property, is a consequence of the decomposition valid for an arbitrary polynomial:

$$
p\left(x_{1}, \ldots, x_{m}\right)-p\left(\lambda_{1}, \ldots, \lambda_{m}\right)=\sum_{j=1}^{m} f_{j}(z)\left(x_{j}-\lambda_{j}\right)
$$

where $f_{i}, 1 \leq i \leq m$, are also polynomials. Let $\lambda \in \sigma_{l}\left(\mathbf{x}_{Z}\right)$, which means that $I_{l}\left(\left\{x_{z}-\lambda(z)\right\}_{z \in Z}\right)$ is a proper left ideal.

For any $y_{u}=p_{u}\left(x_{z_{1}}, \ldots, x_{z_{m_{u}}}\right)$ with all $z_{i} \in Z$ we obtain

$$
B\left(y_{u}-p_{u}\left(\lambda\left(x_{z_{1}}\right), \ldots, \lambda\left(x_{z_{m_{u}}}\right)\right)\right) \subset \sum_{i=1}^{m_{u}} B\left(x_{z_{i}}-\lambda\left(z_{i}\right)\right) \subset I
$$

whence $I_{l}\left(\left\{p_{u}-P(\lambda)(u)\right\}_{u \in U}\right) \subset I$ is a proper ideal. This gives $P(\lambda) \in$ $\sigma_{l}\left(P\left(\mathbf{x}_{Z}\right)\right)$.

In order to prove $\sigma_{l}\left(P\left(\mathbf{x}_{Z}\right)\right) \subset P\left(\sigma_{l}\left(\mathbf{x}_{Z}\right)\right)$, take $\mathbf{y}_{U}=P\left(\mathbf{x}_{Z}\right)$ and $\mu \epsilon$ $\sigma_{l}\left(\mathbf{y}_{U}\right)$. The elements $y_{u}-\mu(u), u \in U$, generate a proper left ideal in $B$.

Set $W=U \cup Z$ and define

$$
s_{w}= \begin{cases}y_{w}, & w \in U \\ x_{w}, & w \in Z\end{cases}
$$

Obviously $\mathbf{s}_{W} \in \mathfrak{c}_{W}(B)$.

The identically zero function on $U$ belongs to $\sigma_{l}\left(\mathbf{y}_{U}-\mu\right)$, so by Theorem 2.1 there exists $\lambda \in \mathbb{C}^{Z}$ such that the elements $y_{u}-\mu(u), u \in U$, and $x_{z}-\lambda(z), z \in Z$, generate a proper ideal $J$. Take any $u \in U$ and let

$$
\begin{aligned}
y_{u} & =p_{u}\left(x_{z_{1}}, \ldots, x_{z_{m_{u}}}\right) \\
& =p_{u}\left(\lambda\left(x_{z_{1}}\right), \ldots, \lambda\left(x_{z_{m_{u}}}\right)\right)+\sum_{j=1}^{m_{u}} f_{j}\left(x_{z_{1}}, \ldots, x_{z_{m_{u}}}\right)\left(x_{z_{j}}-\lambda\left(z_{j}\right)\right) .
\end{aligned}
$$


By the definition of $\lambda$, for all $b_{1}, \ldots, b_{m_{u}} \in B$,

$$
p_{u}\left(x_{z_{1}}, \ldots, x_{z_{m_{u}}}\right)-\mu(u)+\sum_{j=1}^{m_{u}} b_{j}\left(x_{z_{j}}-\lambda\left(z_{j}\right)\right) \in J .
$$

If we choose $b_{j}=-f_{j}\left(x_{z_{1}}, \ldots, x_{z_{m_{u}}}\right)$, we obtain in particular

$$
\left(\mu(u)-p_{u}\left(\lambda\left(x_{z_{1}}\right), \ldots, \lambda\left(x_{z_{m_{u}}}\right)\right)\right) e \in J
$$

which gives $\mu(u)=p_{u}\left(\lambda\left(x_{z_{1}}\right), \ldots, \lambda\left(x_{z_{m_{u}}}\right)\right)$, ending the proof.

Obviously the right ideal versions for the analogously defined right joint spectrum are also valid. If we assume that both left and right maximal ideals are all closed in $B$ then the Harte spectrum defined as

$$
\sigma_{H}\left(\mathbf{x}_{Z}\right)=\sigma_{l}\left(\mathbf{x}_{Z}\right) \cup \sigma_{r}\left(\mathbf{x}_{Z}\right)
$$

has the spectral mapping property.

\section{References}

[1] F. F. Bonsall and J. Duncan, Complete Normed Algebras, Springer, New York, 1973.

[2] H. G. Dales, Banach Algebras and Automatic Continuity, Clarendon Press, Oxford 2000 .

[3] R. E. Harte, Spectral mapping theorem, Proc. Roy. Irish Acad. Sect. A 72 (1972), 89-107.

[4] A. Wawrzyńczyk, Harte theorem for Waelbroeck algebras, Math. Proc. Roy. Irish Acad. Sect. A 105 (2005), 71-77.

[5] W. Żelazko, An axiomatic approach to joint spectra I, Studia Math. 64 (1979), 249261.

Antoni Wawrzyńczyk

Departamento de Matemáticas

Universidad Autónoma Metropolitana - Iztapalapa

Av. San Rafael Atlixco 186, col. Vicentina

AP 55-534

09340 México, D.F., Mexico

E-mail: awaw@xanum.uam.mx

Received October 21, 2006;

received in final form January 10, 2007 